\title{
Mode parameters control under GMAW in connection with reflected heat flow
}

\author{
Anton Melnikov ${ }^{1}$, Mikhail Sholokhov ${ }^{1}$, Darya Buzorina, ${ }^{2,}$, and Andrei Fiveyskiy ${ }^{2}$ \\ ${ }^{1}$ SHTORM Co.Ltd, Ekaterinburg, Russia, 620100 \\ ${ }^{2}$ Federal State Autonomous Educational Institution of Higher Education "Ural Federal University \\ named after the first President of Russia B.N. Yeltsin ", Ekaterinburg, Russia, 620002
}

\begin{abstract}
The present article presents the research results of the heat flow reflection from the edge of the work-piece. Based on the results of a mathematical modeling, we determined the regression equations of the correspondence between the size of the heat reflection zone and the studied parameters and an algorithm for choosing the parameters of the welding mode in the conditions of thermal field distortion due to heat reflection from the edge was developed.
\end{abstract}

One of the tasks under GMAW is to ensure the constancy of the geometric dimensions of the weld throughout the length of the weld, including the end sections, that, according to statistics, are the most defective ones. The reason for this is the postheating of the end of the weld as a result of reflection of the heat flow originating from the heat source from the edge when the heat source approaches it during welding [1-3].

Changing the parameters of the weld seam caused by overheating of the end part weld mostly appears in areas where, due to the structural features of the welded product, it is impossible to install run-off plates on which the end part of the weld seam is extended. Often this applies to cases with the welding of reinforcing ribs in spatial structures (for example, individual modules of bridge spans, etc.). In this regard, the problem arises of choosing the parameters of the welding mode for the end part of the welding joint, as well as determining the dimensions of the heat reflection zone, which would ensure the required quality of the welding joint.

Purpose of work: to develop an algorithm for selecting the mode parameters, that will allow you to take into account the influence of workpiece's edges on the change in the distribution of heat in this area.

Experimental studies of the effect of heat flow reflection from the edge of the workpiece proved that the thermal processes at the end of the welding joint are significantly different from the static pattern observed in the middle of the work-piece. The processes of heat reflection from the edge of the welded plates facilitate overheating of the zone at the end of the weld. Overheating during single-arc welding appear much more significantly (increase in maximum heating temperatures by the end of the sample by 20-35\%), in comparison with double-arc welding, where the increase in maximum heating temperatures by the end of the sample reaches only $10-20 \%$ [4].

\footnotetext{
*Corresponding author: darja.buzorina@yandex.ru
} 
To study further the influence of the heat flow reflection effect and determine the area of its strongest influence, both in single-arc and double-arc welding, as well as to develop algorithms for selecting the mode parameters to reduce its effect, a numerical model of heat distribution processes has been developed taking into account the change in thermophysical properties of material (c $(T), \rho(T), \lambda(T)$ ), which were selected from the reference materials [5].

A moving welding heating source was simulated by the solidus surface of the weld pool, which was set in the form of a double ellipsoid.

The boundary conditions were set in accordance with the scheme shown in Figure 1. The edge of the plate, from which the heat flow is reflected, was set in the form of an adiabatic surface. At other edges, heat transfer to the environment was taken into account.

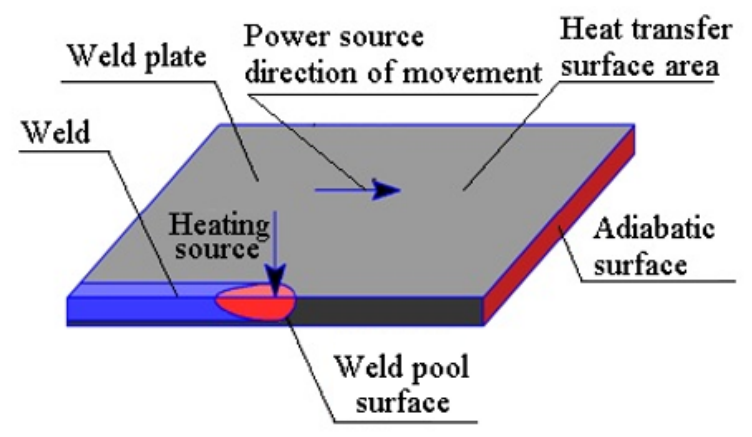

a)

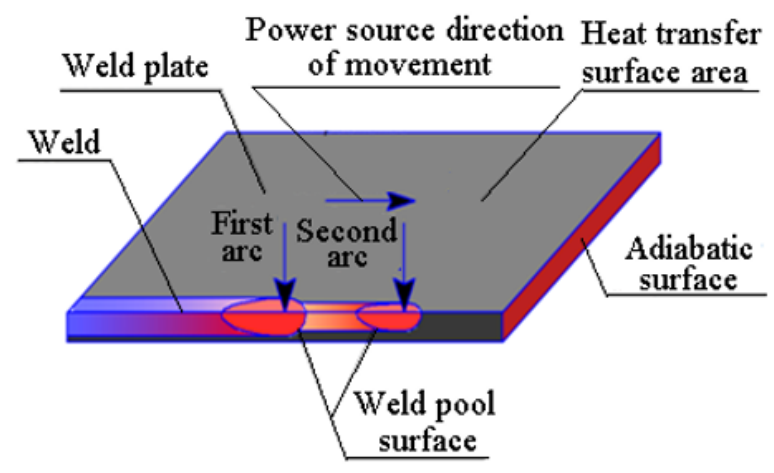

b)

Fig. 1. Scheme for setting of boundary conditions at temperature calculations: a) under single-arc welding; b) under double-arc welding.

The thermal problem was solved by the finite difference method, the grid spacing along the $\mathrm{X}, \mathrm{Y}$, and $\mathrm{Z}$ axes was assumed uniform. To ensure the unconditional stability of the solution, regardless of the time step and coordinate, an implicit scheme was chosen for determining the temperature. When describing the heating and cooling of the work-piece during welding, the method of separation of variables was used, and the sweep method was chosen as a method for solving grid equations.

Based on the developed mathematical model of heat transfer, a computational experiment was conducted to study the influence of the thickness of the weld metal and heat input of the process on the parameters of the area of influence of heat reflection and the change in the width of the weld in this area. 
Based on the results of a computational experiment, regression equations were obtained to determine the length of the overheating area $L_{\text {ref }}$ depending on the metal thickness and heat input and the seam width $e$ in the overheating area depending on the edge distance, metal thickness and heat input:

1) for single-arc welding:

$$
\begin{aligned}
& L_{r e f}=1,78 \cdot q-2,99 \cdot s+43,36 \\
& e=12,68+1,38 \cdot s-3,60 \cdot q-0,13 \cdot l
\end{aligned}
$$

2) for double-arc welding:

- First arc

$$
\begin{aligned}
& L_{r e f 1}=2,50 \cdot q-1,79 \cdot s+27,57 \\
& e=15,32+1,68 \cdot s-11,20 \cdot q-0,03 \cdot l
\end{aligned}
$$

- Second arc

$$
\begin{aligned}
& L_{r e f 2}=2,10 \cdot q-1,47 \cdot s+36,55 \\
& e=14,90+1,51 \cdot s-5,30 \cdot q-0,03 \cdot l
\end{aligned}
$$

The conducted studies allowed us to develop an algorithm for choosing the parameters of the welding mode in the conditions of distortion of the thermal field due to the reflection of heat from the edge.

The choice of welding mode parameters is carried out in several stages. After entering the initial data for the calculation and setting the parameters of the numerical calculation model, the temperature field is calculated using the developed model using the finite difference method. After that, the work-piece is separated along the seam axis into a grid of elements, and the coordinates of the points with the solidus temperature in the separation elements are determined by the results of temperature field calculations.

The length of the welding joint $L$ is divided along its axis into $n$ - the number of elements with the same pitch $\Delta x$. The number of steps of the separation $n$ is determined in accordance with the following correspondence:

$$
n=L / \Delta x
$$

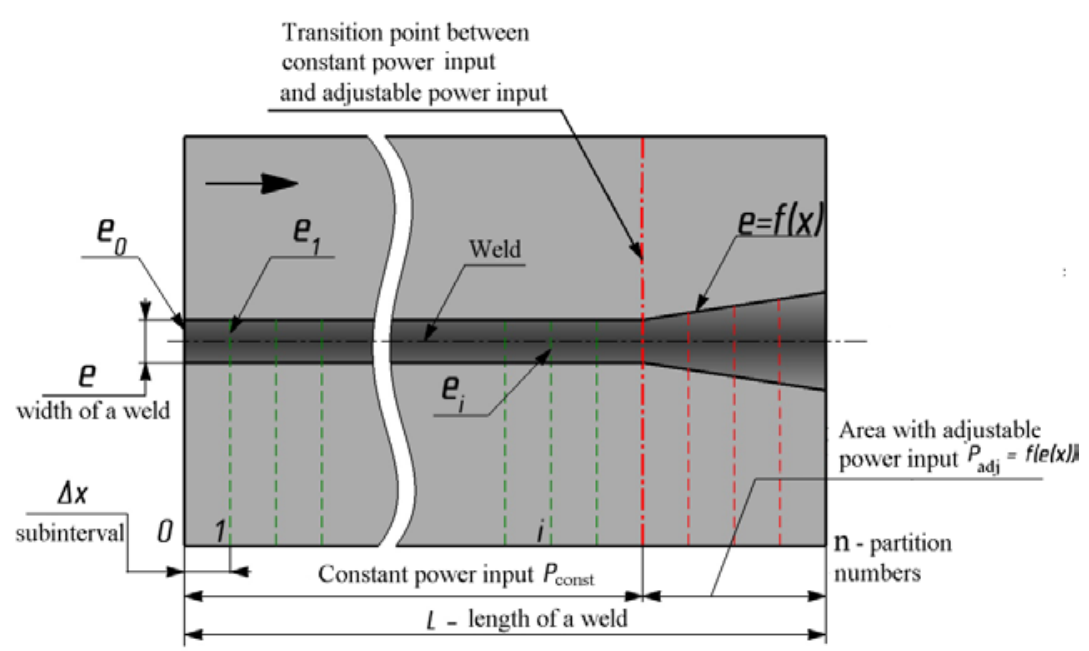

Fig. 2. Weld length separation scheme for determining the control function. 
Then an array of point positions with a solidus temperature is formed $M=\left\{\mathrm{x}_{\mathrm{i}} ; \mathrm{y}_{\mathrm{i}}\right\}$, at (T $\left.=\mathrm{T}_{\text {sol }}\right) \rightarrow e_{i}(x)$, where $e_{i}$ is the width of the weld along the $x$ coordinate; $e_{i}=2 y_{i}$.

After that, sequential comparison of the actual width of the weld in each element of the separation with a standardized value is performed:

$$
\Delta_{\mathrm{i}}=\left|e_{i}(x)-e_{\text {standard }}\right|>\Delta_{\text {standard }}
$$

If the standardized value is exceeded, the heat input of the process is adjusted:

$$
q_{\text {кор } i}=q_{\text {пост }} \frac{\mathrm{e}_{\text {standard }}}{e_{i}(x)}
$$

At the end of the calculation cycle an array of coordinate values and corresponding arc powers is formed $\Omega=\left\{\mathrm{x}_{\mathrm{i}} ; \mathrm{Q}\right.$ кор $\}$.

At the last stages, current and voltage control functions are formed on the basis of the array $\Omega$.

for single-arc welding $-\left\{\begin{array}{l}I=f(x) \\ U=f(x)\end{array}\right.$, under condition $q(x)=\frac{\eta \cdot I(x) \cdot U(x)}{V_{c B}}$

for double-arc welding $-\left\{\begin{array}{l}I_{1}=f_{1}(x) ; U_{1}=f_{1}(x) \\ I_{2}=f_{2}(x) ; U_{2}=f_{2}(x)\end{array}\right.$,

under condition $\left\{\begin{array}{l}q_{1}(x)=\frac{\eta \cdot I_{1}(x) \cdot U_{1}(x)}{V_{c B}} \\ q_{2}(x)=\frac{\eta \cdot I_{2}(x) \cdot U_{2}(x)}{V_{c B}}\end{array}\right.$

The function for controlling the parameters of the welding mode is determined, which is transmitted to the actuators (robotics and automation) and the control system of the actuator generates a welding program based on the choice function of the parameter mode, where the calculated values of I and U correspond to each x coordinate. Welding is performed.

The application of the developed algorithm in test welding performed on a robotic complex made it possible to provide the required parameters of the welding joint: width of a weld, depth of penetration, welding thermal cycle, constant (unchanged) HAZ structure along the entire weld, including the end of the weld [8].

Based on experimental and theoretical studies, including the data of a computational experiment, it was possible to determine the areas of effective application of the developed algorithm for single-arc and double-arc welding of low alloy structural steels with material thicknesses from 2 to $14 \mathrm{~mm}$. (Figure 3) [8].

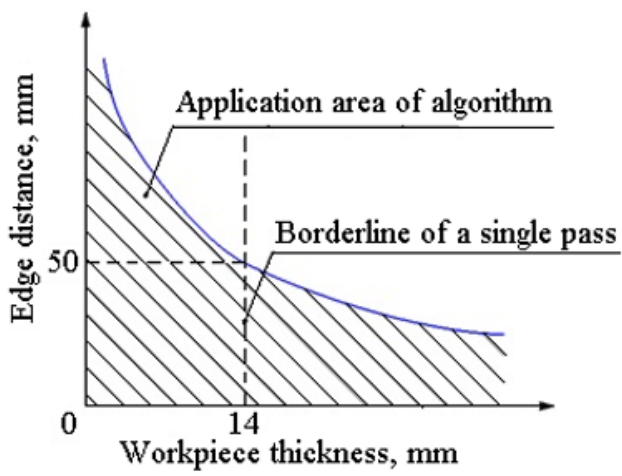

a)

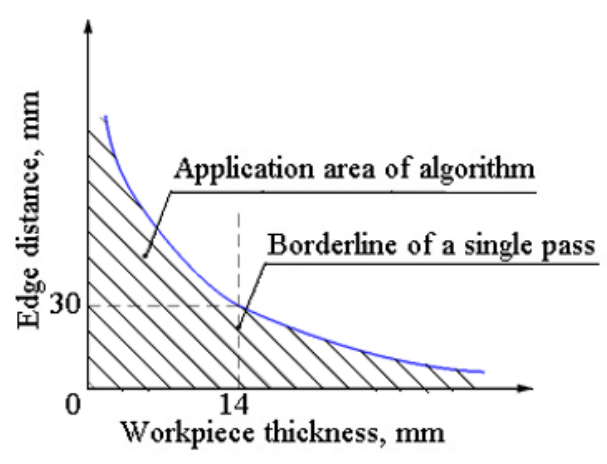

b)

Fig. 3. Areas of effective application of the welding mode selection algorithm: a) under single-arc welding for the process speed from 10 to $20 \mathrm{~m} / \mathrm{h}$; b) under double-arc welding for the process speed from 20 to $30 \mathrm{~m} / \mathrm{h}$ 
The welding mode selection algorithm is to be applied most effectively in a single-pass welding, that is, in the absence of additional heat treatment by subsequent passes, for thicknesses of the work-pieces to be welded up to $14 \mathrm{~mm}$. The algorithm developed in this work was also successfully implemented during automatic double electrode gas metal arc welding of complex spatial building structures for welds in which it is impossible to install run-off plates.To implement this technology, a double-arc welding machine (welding tractor) ADFG-502 SHTORM was developed, the design features of which were protected by a patent $[6,7]$.

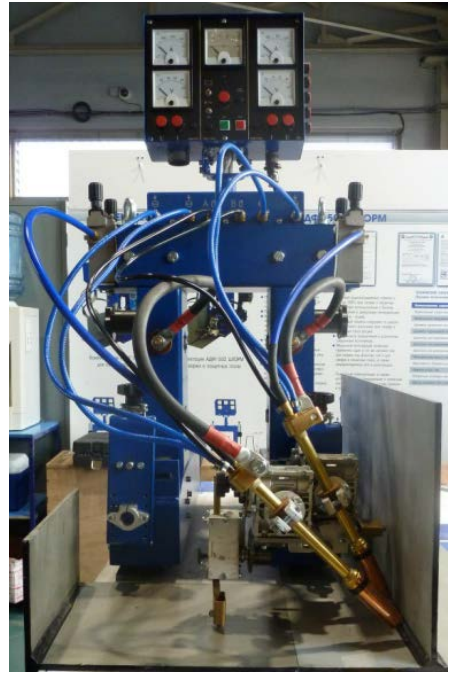

a)

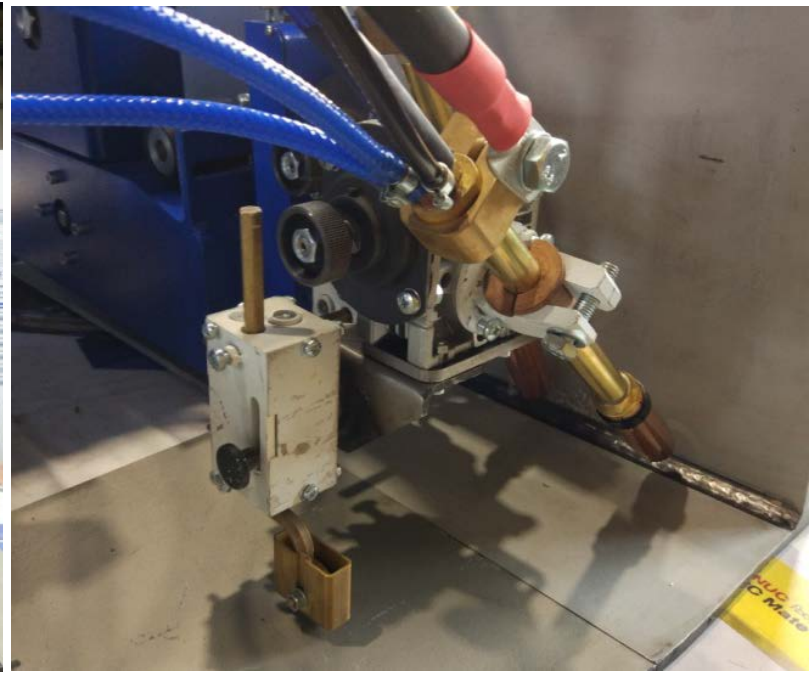

b)

Fig. 4. A welding machine equipped with a unit for attachment of torches for double-arc welding: a) general view; b) unit for attachment of torches

In accordance with the model developed in this work, the control function of parameters (thermal power input) for double-arc welding was determined in accordance with the target function to ensure the required standard dimensions of the weld (Figure 5).

The introduction of the ADFG-502 SHTORM automatic welding machine into production made it possible to increase the productivity of the welding process by 1.5-2.0 times in comparison with the use of single-arc processes, as well as reduce the number of defective products by $15-20 \%$. Implementation results are reflected in the Implementation Act. 


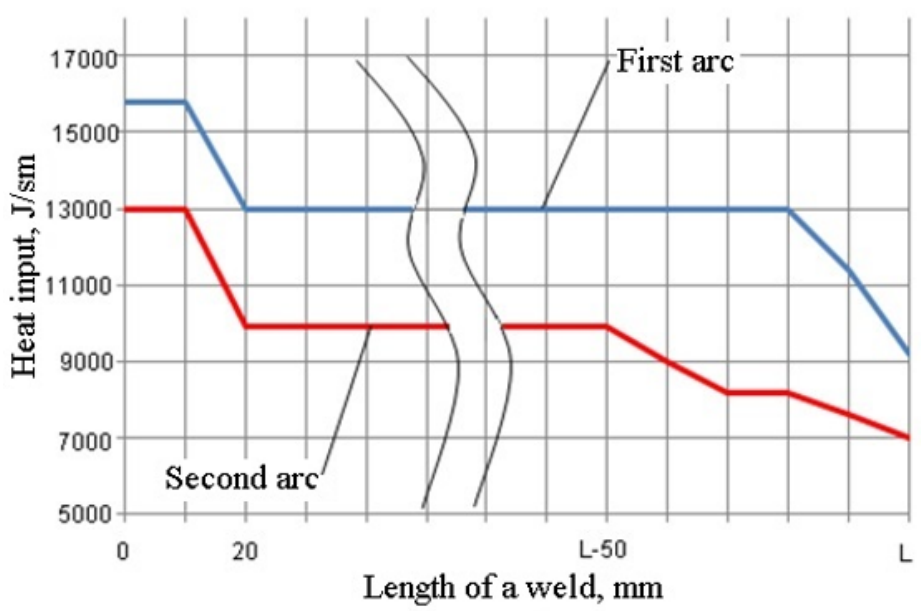

a)

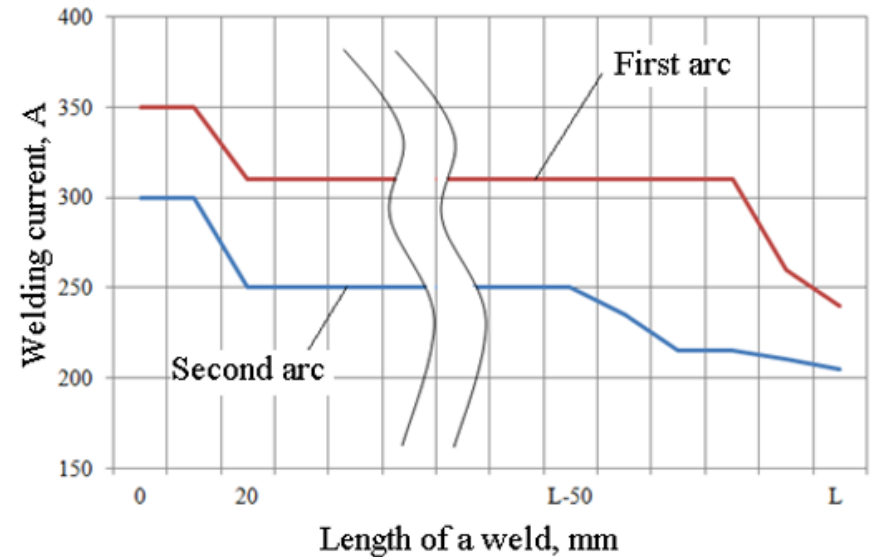

b)

Fig. 5. Distribution of energy parameters of the first and second arc along the weld: a) heat input; b) welding current

\section{Conclusions}

1. A mathematical model of heat transfer has been developed, which, in addition to taking into account changes in the thermophysical properties of the welding joint and the distribution of the heat source, has also described the reflection of heat from the edge. Based on the results of experimental studies and modeling of thermal processes, an algorithm was developed for choosing the welding mode under the influence of reflected heat flux, which was used to create welding cells.

2. Areas of effective application of the developed algorithm for single-arc and doublearc single-pass welding for thicknesses of the work-pieces from 2 to $14 \mathrm{~mm}$ are determined.

3. The introduction of the developed algorithm for choosing the welding mode allowed us to significantly improve the quality of welding joints with a reduction in the number of defective products by $20 \%$, to ensure the stability of the geometric dimensions of the weld along the entire length of the welding joint. The increase in productivity in single-arc welding of circular seams is $50 \%$, in double-arc welding of structures without run-off plates - 50-100\%. This algorithm can be used as part of software in automatic and robotic units. 


\section{References}

1. Karhin V.A.: Thermal processes in welding (2nd ed.) (SPb.: Publishing house of Polytechnic. Univ., 2015)

2. V.S. Ageev The causes of cracks orthotropic steel decks. Transportnoye Stroitelstvo v.11, pp. 18-20 (2002)

3. A.P.Gulyaev Impact hardness and cold brittleness of structural steel. ( Mashinostroyeniye, 1967).

4. M.A. Sholokhov, A.Y. Melnikov, D.S. Buzorina. Research of the effect of reflected heat flow on the change in the characteristics of the end part of the weld. Svarka \& Diagnostika, v.5. pp. 30-37 (2018)

5. M.A. Sholokhov, A.Y. Melnikov, D.S. Buzorina Improvement of modeling of heat distribution processes under boundary constraint conditionas of work-piece. Svarka \& Diagnostika, v. 6, pp. 33-39 (2018)

6. Utility Model Patent No.139365 (RF) Double Arc Welding Machine. Sholokhov M.A., Fiveiskii A.M., Matushkin A.V., Melnikov A.Y.; application dd 08.03.2013; published 17.03.2014. Ticket No. 11.

7. Utility Model Patent No.144972 (RF) Torch positioning unit. Sholokhov M.A., Fiveiskii A.M., Matushkin A.V., Melnikov A.Y.; application dd 25.04.2014; published 10.09.2014. Ticket No. 25.

8. A.Y. Melnikov, M.A. Sholokhov, D.S. Buzorina, A.E. Filyakov Application of mathematical modeling methods to study the influence of technological parameters under arc welding on the dimensions of the heat reflection zone (to be published) 